\title{
Early ultrastructural changes of antral mucosa with aspirin in the absence of Helicobacter pylori
}

\author{
C J McCarthy, E Sweeney, C O'Morain
}

\begin{abstract}
Aims-To describe the ultrastructural changes that occur in human antral mucosa following direct application of aspirin in volunteers without Helicobacter pylori infection.

Methods-Ten healthy male volunteers without $H$ pylori infection underwent three consecutive endoscopies (at zero, one and five hours). At the first endoscopy, two biopsy specimens were obtained (one for histology and the other for electron microscopy (EM)). At subsequent endoscopies, a single biopsy specimen was obtained for EM. A $50 \mathrm{ml}$ solution of aspirin (concentration $3 \mathrm{mg} / \mathrm{ml}$ ) was applied to the antral mucosa at the first endoscopy in five subjects; the other five subjects received 50 $\mathrm{ml}$ distilled water (placebo).

Results-The ultrastructural appearance of the first biopsy specimen in all subjects and subsequent biopsy specimens in the placebo treated subjects was normal. The aspirin treated group had evidence of intercellular oedema, widening of capillary fenestrae, rupturing of apical membranes, and dilatation of endoplasmic reticulum and mitochondria after one hour; these changes were more marked at five hours. Tight junctions were maintained.

Conclusion-This is the first study to describe the early ultrastructural changes in antral mucosa induced by aspirin in subjects without $H$ pylori infection.

(F Clin Pathol 1995;48:994-997)
\end{abstract}

Keywords: Helicobacter pylori, antral mucosa, aspirin.
The early ultrastructural changes that occur in gastric and duodenal mucosa following the administration of non-steroidal anti-inflammatory drugs (NSAIDs) have been studied in humans and animals. ${ }^{1-6}$ None of these studies, however, evaluated the changes that occur in the absence of Helicobacter pylori infection. $H$ pylori is found in close contact with the epithelial cells of the antral mucosa, and is associated with ultrastructural changes including cell necrosis and autolytic intracytoplasmic vacuoles. ${ }^{78}$ As $H$ pylori infection is common, the true nature of the ultrastructural changes of antral mucosa that occur with NSAIDs in the absence of $H$ pylori infection have not been described.

The aim of this study was to assess the ultrastructural changes that occur in human antral mucosa, over a five hour period, following direct application of aspirin to the antral mucosa in volunteers. Only those subjects who were negative for $H$ pylori infection were included in this study.

\section{Methods}

Ten healthy male subjects with a mean age of 29 (range 26-32) years were studied. Seventeen subjects were initially screened for the presence of $H$ pylori infection with a ${ }^{13} \mathrm{C}$ urea breath test. Seven were excluded due to a positive ${ }^{13} \mathrm{C}$ urea breath test. The ${ }^{13} \mathrm{C}$ urea breath test was carried out according to the European Standard Protocol, ${ }^{9}$ which has a sensitivity and specificity of $99 \%$ and $98 \%$, respectively. One antral biopsy specimen was taken for histological evaluation to ensure that $H$ pylori was not present.

Accepted for publication 1 June 1995

Figure 1 Features of normal antral mucosal epithelium. (A) Low power view showing apical mucin vacuoles, stubby surface microvilli and complexly interleaved basilateral cell membranes (original magnification, $\times 1500$ ). (B) High power view of an intercellular junction. $O=z o n u l a$ occludens; $A=$ zonula adherens. Three maculae adherens are indicated by arrows (original magnification, $\times 9000$ ). (C) Detail of cytoplasmic organelles including mitochondria with lamellar cristae, Golgi apparatus $(G)$ and numerous free ribosomes (original magnification, $\times 9000)$. 


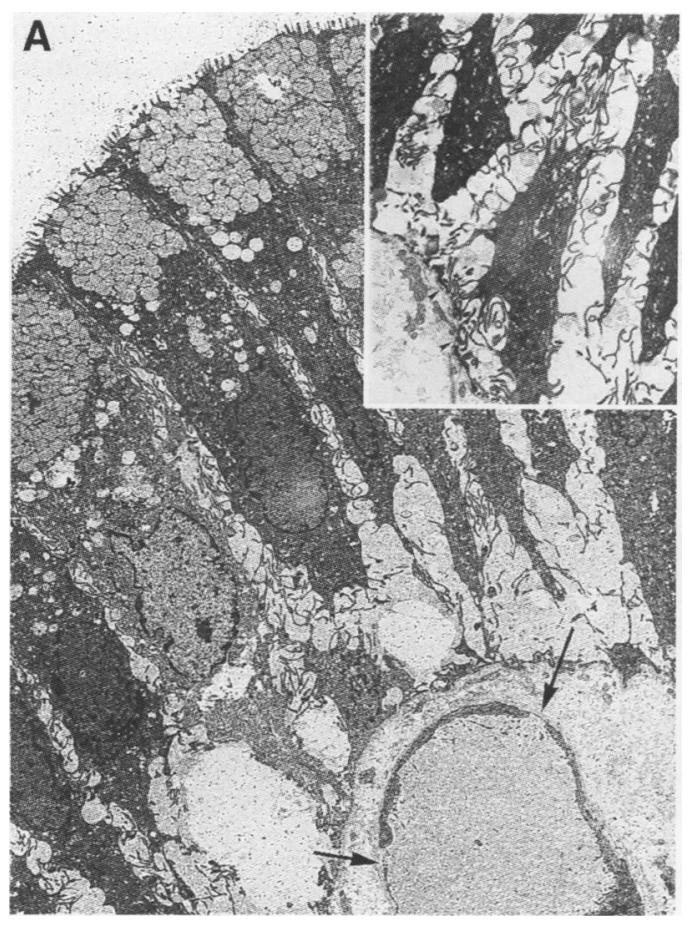

Figure 2 One hour after aspirin treatment. (A) There is pronounced oedema between the basilateral aspects of the epithelial cells. A capillary in the lamina propria (bottom right) contains granular appearing plasma and exhibits widening of endothelial cytoplasmic fenestrae (arrows; original magnification, $\times 1500)$. Inset: high power view of basilateral folds of cell membranes widely separated by oedema fluid. Arrowheads indicate basal lamina (original magnification, $\times 4000)$. (B) In another case, in addition to intercellular oedema, there is rupture of the apical membrane of an epithelial cell and sludging of red blood cells (bottom and right) in distended lamina propria capillaries (original magnification, $\times 1500$ ).

Other than $H$ pylori infection, exclusion criteria included a previous history of peptic ulcer disease, smoking, recent antibiotic use, and habitual use of NSAIDs.

The study was approved by the local research ethics committee and informed consent was obtained from all subjects.

Each subject underwent three endoscopies at time zero, one and five hours. Antral biopsy specimens were taken at each endoscopy; two at the first endoscopy and one at the second and third, respectively. The antral area was considered to have three equal segments with the pylorus as the centre point. Biopsy specimens were taken from different segments at each endoscopy.

\section{ENDOSCOPY 1}

The mucosa was assessed visually to ensure the presence of a normal mucosa. Two antral biopsy specimens were taken from one segment of the antral mucosa, the first for histological evaluation and the second for electron microscopic (EM) evaluation. The biopsy specimen for histology was transported in 5\% formaldehyde, and that for EM in $4 \%$ glutaraldehyde. A $50 \mathrm{ml}$ solution of aspirin (concentration $3 \mathrm{mg} / \mathrm{ml}$ ) was applied directly to the antral area, using a cannula, in five subjects; $50 \mathrm{ml}$ distilled water (placebo) was applied to the antral mucosa in five other subjects.

\section{ENDOSCOPY 2}

At this endoscopy, one hour after the first, a single biopsy specimen was taken from a second segment of the antral mucosa for EM evaluation. control subjects were normal with no evidence of ultrastructural change. The ultrastructural appearances of the antral mucosa one hour after application of aspirin directly to the antral

mucosa are shown in fig 2 . In the aspirin treated

\section{Results}

Histological assessment of each antral biopsy specimen was normal, with no evidence of $H$

The ultrastructural appearances of normal antral mucosa in the entry biopsy specimens are complexly interleaving basilatera cell membranes. The ultrastructural appearances of each of the entry biopsy specimens were regarded as normal.

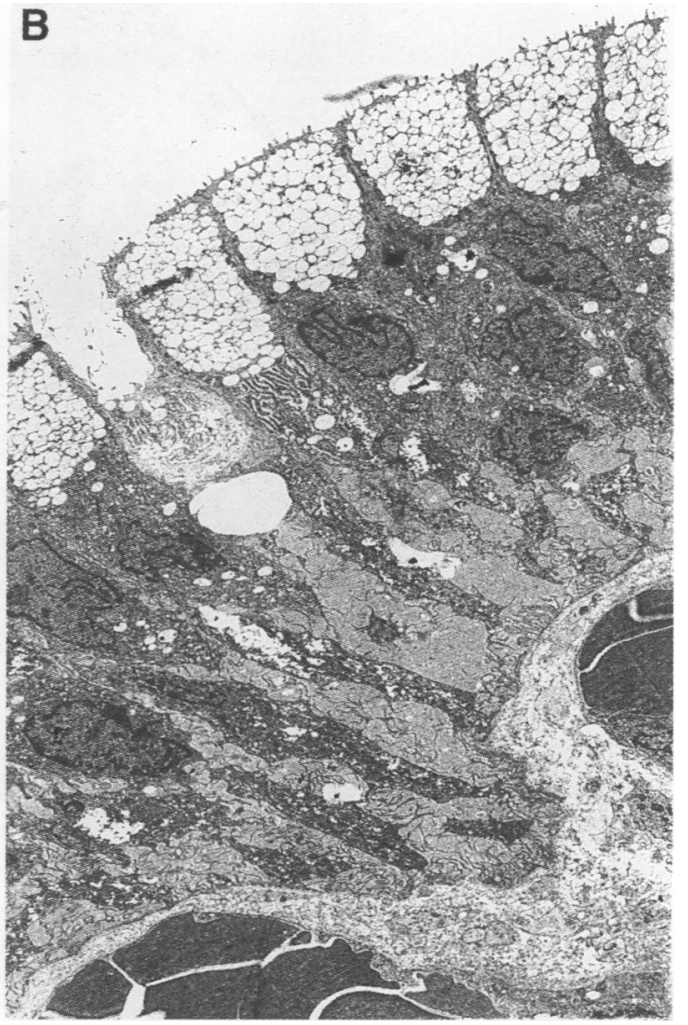




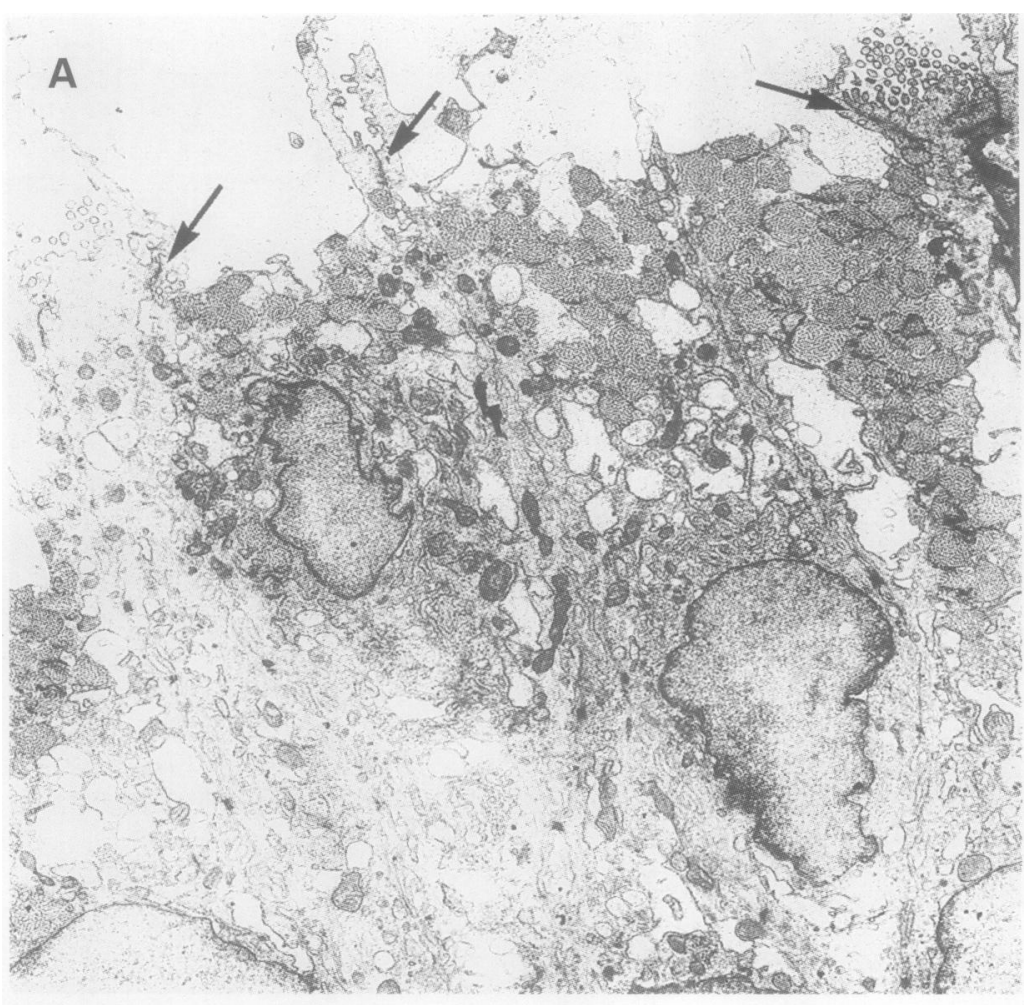

Figure $3(A)$

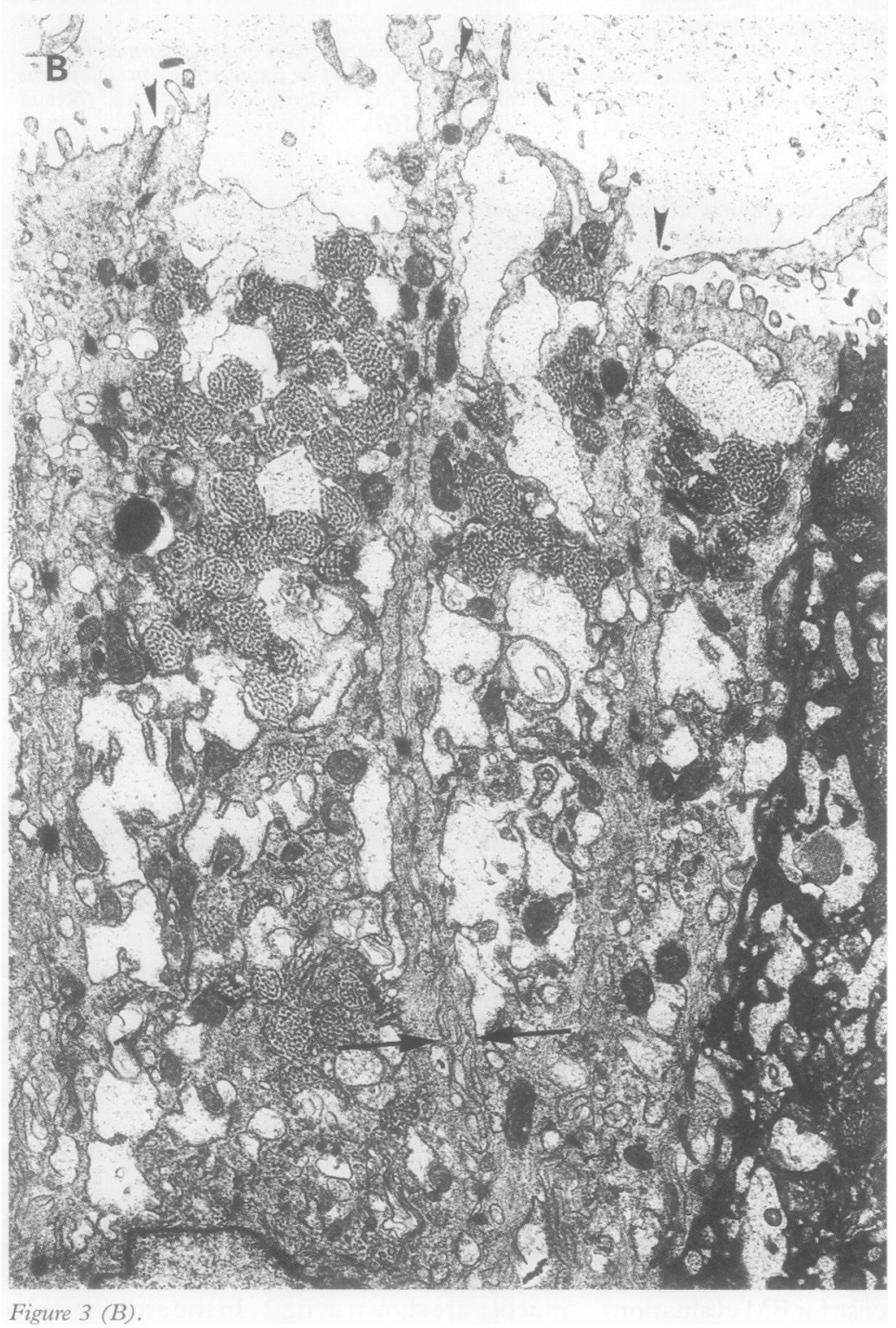

group changes from normal were evident in all of the specimens, including intercellular oedema, widening of the fenestrae of the capillaries, rupturing of the apical membranes, and dilatation of the endoplasmic reticulum. The tight junctions were maintained.

Five hours after the application of aspirin, marked changes were seen in the epithelium of the test cases (fig 3), and included extensive rupture of apical cell membranes, dilatation of endoplasmic reticulum and swelling with loss of cristae in mitochondria. Tight junctions were retained. There was a noticeable increase in the number of epithelial cells showing both nuclear and cytoplasmic condensation.

In summary, in control subjects there was no evidence of ultrastructural changes at zero, one or five hours. In the aspirin treated group all entry biopsy specimens were normal, but there were changes in all the subsequent specimens taken at one hour and these changes were more marked at five hours.

\section{Discussion}

This study is the first to report the ultrastructural changes that occur in the antral mucosa following the direct application of aspirin to the mucosa in subjects in whom $H$ pylori infection is absent. These changes include widening of the endothelial cytoplasmic fenestrae, intercellular oedema and separation of the folds of the basilateral cell membranes, swelling of mitochondria and endoplasmic reticulum, rupture of the apical surface membranes with mucin discharge, and cellular degeneration. Interestingly, as in previous studies, ${ }^{12}$ the tight junctions of the cells remained intact even with marked destruction of cells.

This study included a placebo limb, because of the possibility that these changes could be induced by taking the biopsy specimen-that is, from antral bleeding and a release of inflammatory mediators. A previous study ${ }^{4}$ reported that benoxaprofen produced less ultrastructural changes than aspirin in rat and pig gastric mucosa. It was postulated that benoxaprofen caused less extravasation of blood and thereby lead to reduced ultrastructural change. However, the results reported here suggest that bleeding does not play a significant role in the ultrastructural changes that occur with NSAIDs.

Baskin $e t a l^{2}$ were the first group to study the ultrastructural changes in human gastric mucosa after the direct application of routine doses of aspirin. They reported damage in all biopsy specimens after aspirin application. Peak damage occurred after 10 minutes, with $25 \%$ of the surface epithelial cells showing some evidence of damage, while at one hour there was a decrease in the extent of focal damage and recovery of most of the mucosa. Only one subject was biopsied at six hours and this specimen showed almost complete recovery. In the present study damage was maximal at five hours. In the study by Baskin $e t a l^{2}$ the aspirin was applied via nasogastric tube which was placed in the gastric fundus under fluoroscopic control, and the biopsy specimens were taken 


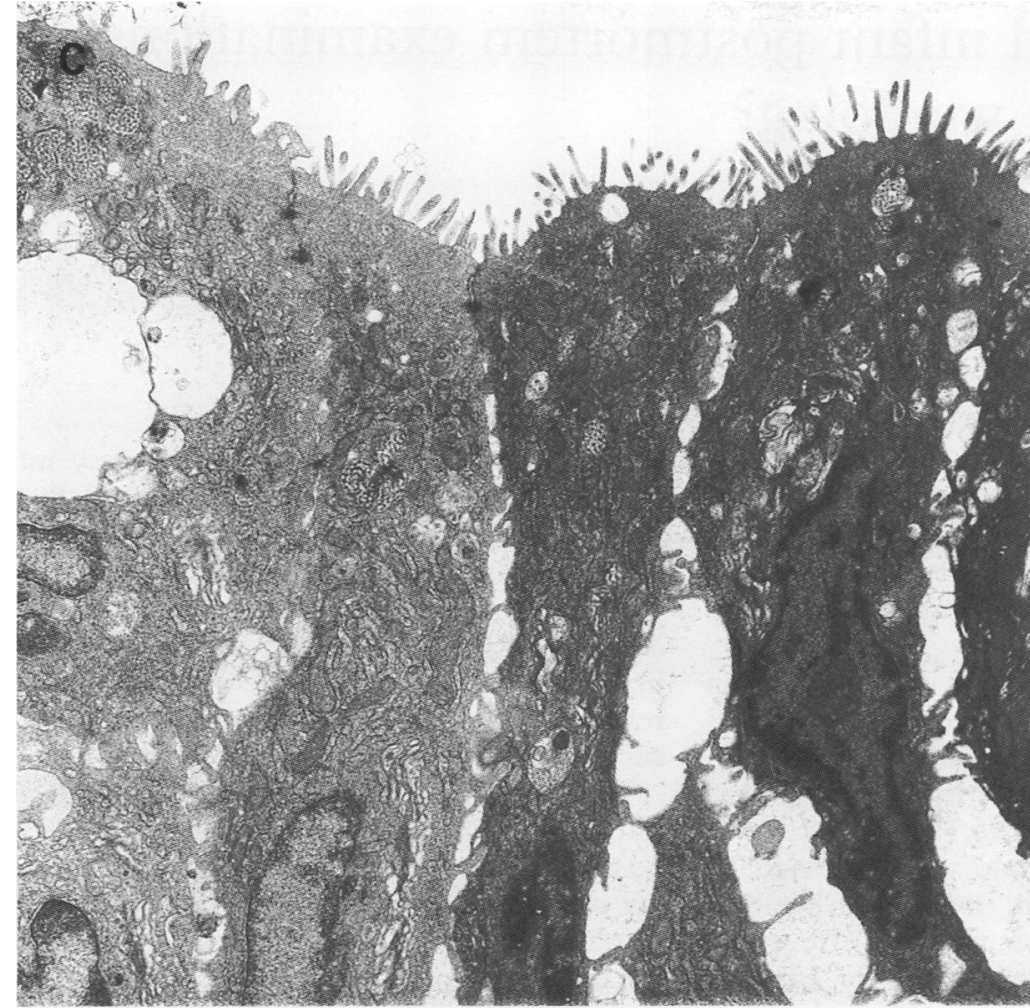

Figure $3(C)$

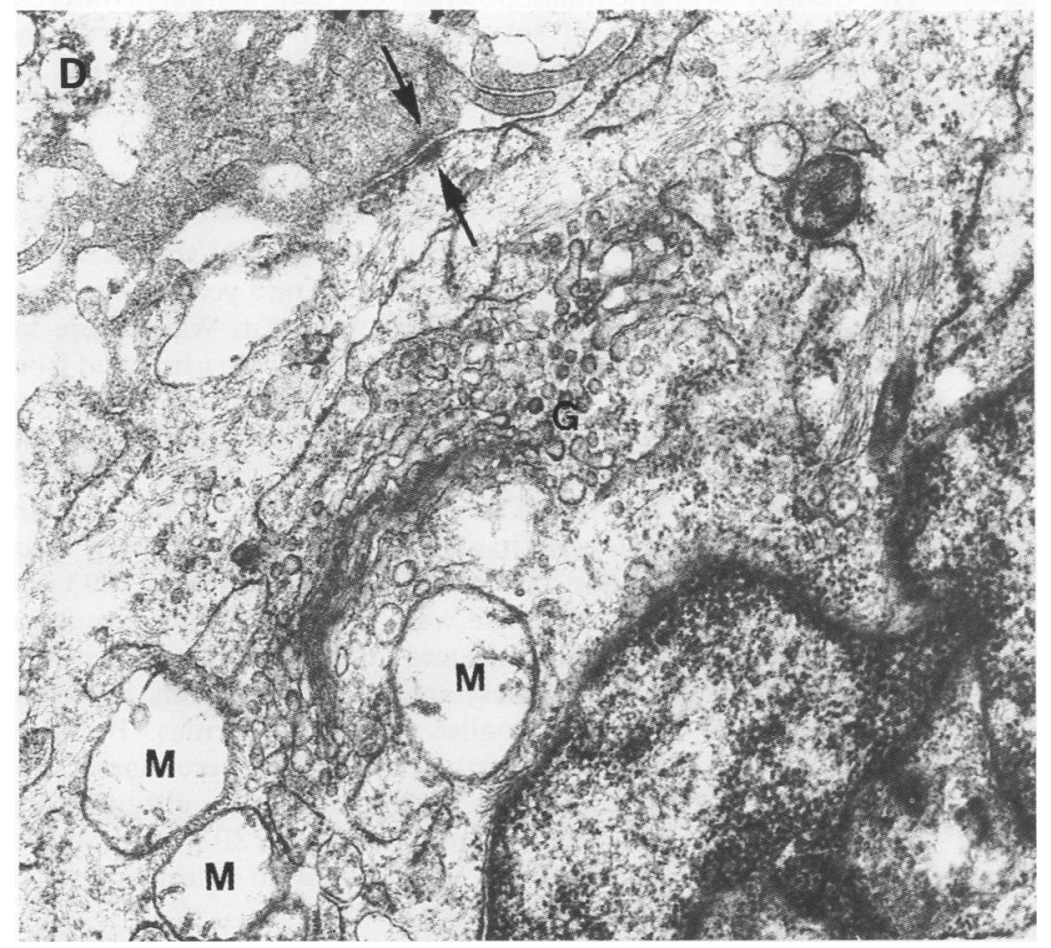

Figure 3 Five hours after aspirin treatment. (A) There is evidence of rupture of apical membranes in several contiguous epithelial cells. Tight junctions (arrows) are retained (original magnification, $\times 2000$ ). (B) $A$ higher power view shows prominent dilatation of endoplasmic reticulum, partially ruptured apical membranes and preserved tight junctions (arrowheads). Intercellular oedema is not evident in this case at this level (high luminal aspect of cells) and the normal close interleaving of lateral cell membranes is still seen (arrows; original magnification, $\times 3500)$. (C) In another case oedema extends upwards to the level of tight junctions between three darkly staining condensed epithelial cells. Similar cells were seen infrequently in control biopsy specimens. The apical portion of a cell (left) contains large blebs, possibly grossly distended endoplasmic reticulum (original magnification, $\times 3000)$. (D) Mitochondria (M) exhibit prominent swelling with only tags of cristae remaining. The Golgi apparatus $(G)$ is normal and the tight junction (arrows) is preserved (original magnification, $\times 12000$ ). via the nasogastric tube. However, in this study the aspirin was applied and biopsy specimens were taken with direct visualisation of the antral mucosa. This is important to ensure, firstly, that specimens are taken from normal appearing mucosa and, secondly, that they are taken from an area where the aspirin had been applied.

In $1980^{3}$ a report suggested that the rat gastric mucosa responded to aspirin by dilatation of the small vessels of the mucosa with eventual rupture of the endothelial cells. These dilatations are followed by changes in the mitochondria, the organelles which are most sensitive to oxygen deprivation. Aspirin uncouples oxidative phosphorylation ${ }^{1011}$ and causes rapid osmotic swelling of isolated mitochondria. ${ }^{12}$ Aspirin may damage the gastric mucosal in this way. ${ }^{1314}$ Vascular changes were evident in this study, primarily with an increase in the size of the endothelial fenestrae. It is difficult to postulate what is the first change to occur at an ultrastructural level, although the intercellular oedema with rupture of the apical membranes seems to be the first event to occur.

This study reports the changes that occur in the antral mucosa one and five hours after the application of aspirin to the mucosa in healthy male subjects without $H$ pylori infection. Further ultrastructural studies in subjects with $H$ pylori infection are needed to show whether there are important differences from the changes reported here.

We would like to thank Carol McCormick and Ann Myers in the Department of Pathology for their help with this study.

1 Hingson JD, Ito S. Effect of aspirin and related compounds on the fine structure of mouse gastric mucosa Gastroentemlogy 1971:61:156-77.

2 Baskin WN, Ivey KJ, Krause WJ, Jeffrey GE, Gemmell RT. Aspirin-induced ultrastructural changes in human gastric Aspirin-induced ultrastructural changes in

3 Robins PG. Ultrastructural observations on the pathogenesis of aspirin-induced gastric erosions. Br $\mathcal{F}$ Exp Pathol 1980 61:497-504.

4 Rainsford KD, Willis CM, Walker SA, Robins PG. Electron microscopic observations comparing the gastric mucosal damage induced in rats and pigs by benoxaprofen and aspirin, reflecting their differing actions as prostaglandin synthesis inhibitors. Br f Exp Pathol 1982;63:25-34

5 Meschter CL, Gilbert M, Krook L, Maylin G, Corradino $R$. The effects of phenylbutazone on the intestinal mucosa of the horse: a morphological, ultrastructural and biochemical study. Equine Vet 7 1990;22:255-63.

6 Hayllar J, Somasundaram S, Sarathchandra P, Levi AJ, Bjarnason I. Early cellular events in the pathogenesis of NSAID enteropathy in the rat [abstract]. Gastrenterology 1991;100:A216.

7 Tricottet V, Bruneval P, Vire O, Camilleri JP. Campylobacter-like organisms and surface epithelium abnormalities in active chronic gastritis: an ultrastructural study. Ultrastruct Pathol 1986;10:113-22.

8 Kazi JL, Sinniah R, Zaman V, Ng ML, Jafarey NA, Alam SM, et al. Ultrastructural study of Helicobacter pyloriassociated gastritis. F Pathol 1990;161:65-70.

9 Logan RPH, Dill S, Bauer FE, Walker MM, Hirschl AM, Gummett PA, et al. The European Standard ${ }^{13} \mathrm{C}$-urea breath test for the detection of Helicobacter pylori. Eur $\mathcal{F}$ Gastroenterol Hepatol 1991;3:915-21.

10 Haas R, Parker WD, Stumpf D, Eguren LA. Salicylateinduced loose coupling: protonmotive force measurements. Biochem Pharmacol 1985;34:900-2.

11 Whitehouse MW. Biochemical properties of antiinflammatory drugs: III. Uncoupling of oxidative phosphorylation in a connective tissue (cartilage) and live mitochondria by salicylate analogues. Biochem Pharmacol 1964;13:319-36.

12 You K. Salicylate and mitochondrial injury in Reye's syndrome. Science 1983;221:163-5.

13 Kauffman G. Aspirin-induced gastric mucosal injury: Lessons learned from animal models. Gastroenterology 1989; 96:606-14.

14 Gutknecht J. Salicylates and proton transport through lipid bilayer membranes: a model for salicylate-induced uncoupling and swelling in mitochondria. F Membrane Biol 1990;115:253-60. 